\section{Angled eccentric osteosynthesis, a new technique for simple mandibular fractures}

\author{
Jeong Keun Lee \\ Department of Dentistry, Ajou University \\ School of Medicine, Suwon, Korea
}

\section{Abstract}

Working and balancing sides are subject to torsional and the tensile stresses, respectively. Tension banding principle denotes the principle of plating along the tension line for stable internal fixation on the balancing side of the mandibular fracture, which is the theoretical basis for the miniplate osteosynthesis. On the contrary, Arbeitsgemeinschaft für 0steosynthesefragen/Association for the Study of Internal Fixation (A0/ASIF) introduced the idea of stable fixation which confirms the stability of the fracture segment across the fracture line of the working side. Merging the principles of the miniplate osteosynthesis and compression osteosynthesis, the author suggests a new technique of angled eccentric osteosynthesis in case of simple mandibular fractures. The drilling holes are eccentrically positioned away from the fracture gap and intentionally angled to each other. The advancement of the miniscrews through these eccentrically angled drill holes will exert an axial load toward the fracture gap providing an interfragmental compressive force.

\section{Introduction}

In the treatment planning of the mandibular fractures, there are two schools of plate osteosynthesis group, one advocating compression osteosynthesis and the other favouring miniplate osteosynthesis. Arbeitagemeinschaft for 0steosynthesefragen/Association for the Study of Internal Fixation (AO/ASIF) introduced the idea of axial compression into the limb fractures. As applied in the mandible, the idea of compression osteosynthesis was fortified with Dynamic Compression Plate (DCP) and Eccentric DCP in addition to the concept of load bearing plates. ${ }^{1-4}$

Fractured fragments must be stably fixed and not allowed to move because primary bone repair only occurs under conditions of mechanical rest. Regarding the biomechanics of the facial skeleton during mastication, stress vectors of the midface are mainly compressive force along the vertical trajectories, whereas those of the mandible are chiefly bending and twisting. In the functional movement, the balancing side of the mandible is subject to bending which exerts tensile force on alveolar part of the mandible. ${ }^{5}$ Tension banding principle denotes the principle of plating along this tension line for stable internal fixation of the mandibular fracture, which is the theoretical basis for the miniplate osteosynthesis. ${ }^{6}$

Contrary to balancing side, the working side of the functioning mandible is subject to twisting forces causing torsional stress which must be neutralized by some powerful load-bearing plate. Also called as reconstruction plate, it is applied to buttress the comminuted fractures (as a buttressing plate) or to bridge the gaps of the segmental defect caused by major facial trauma or ablative tumour surgery (as a bridging plate).

Originally designed by AO/ASIF for limb surgery, ${ }^{2}$ the DCP was modified by Spiessl to match the mandibular surgery. ${ }^{7}$ In principle, the recesses for the screws should be located at the far ends of the plate holes to be approximated by the effect of the spherically shaped holes when tightened. The perpendicular force delivered during screwing is transferred axially via the spherical screw head and plate hole, which plays as an approximating force.

In a case of simple mandibular fracture the fragments are stable across the gap and both fragments are self-buttressed to each other. Plate osteosynthesis is therefore sufficient with load sharing type. But the principle of interfragmental compression is another buttress to the stable internal fixation in addition to the tension banding principle. We resort to monocortical miniplate osteosynthesis with adoption of the idea of compression osteosynthesis. Conventional miniplate osteosynthesis system was to be found to have interfragmental compressive action with some minor technical modification. Our experiences with miniplate osteosynthesis system were similar to those with DCP of above mentioned.

\section{Technique}

Initially, the drilling hole is eccentrically positioned away from the fracture gap as in Figure 1. Miniscrews have a gap or break with the inner hole of a miniplate. Using this gap, the drilling holes across the fracture gap is intentionally angled to each other as Figure 2. Miniscrews are advanced beginning in the eccentric hole with some degree to the true perpendicular direction. The advancement of the miniscrews along these carefully prepared holes will exert an axial load toward the fracture gap providing an interfragmental compressive force.

\section{Clinical experience}

A 25-year-old man presented to emergency
Correspondence: Jeong Keun Lee, San 5 Wonchon-dong, Yeongtong-gu, Suwon 443-721, Korea (R.O.K.).

Tel. +82.31.219.5333 - Fax: +82.31.219.5329.

E-mail: arcady@ajou.ac.kr

Key words: angled eccentric osteosynthesis, miniplate osteosynthesis, compression osteosynthesis.

Conflict of interest: the authors report no conflicts of interest.

Received for publication: 30 January 2011.

Revision received: 22 February 2011.

This work is licensed under a Creative Commons Attribution 3.0 License (by-nc 3.0).

@C Copyright J.K. Lee, 2011

Licensee PAGEPress, Italy

Surgical Techniques Development 2011; 1:e1 doi:10.4081/std.2011.e1

room with a chief complaint of traumatic injury on his lower facial area. Initial panoramic radiograph revealed occlusal step between fracture segments at the preoperative panoramic radiograph. Two days of elastic traction with Archbar improved the occlusion with no occlusal step between the fracture segments at the immediate preoperative panoramic radiograph.

Gap was identified at the initial surgical access to the fracture site which was tightened with 2 miniplates across the fracture gap utilizing the above-mentioned method. After the application of the angled eccentric osteosynthesis technique minimised gap was identified in Figure 3, which evidenced the interfragmental compression effect with miniplate osteosynthesis system.

\section{Discussion}

At the very moment the fracture occurs begins wound healing process. If there is no deviation and fracture was rigidly, anatomically reduced with less than $0.1 \mathrm{~mm}$ gap, contact repair follows. ${ }^{1}$ The fracture line is closely adapted and compre ssed so there is no displacement at the fracture site. Histologically the shape of the osteones does not change crossing the fracture line. Initial lamellar bone deposition is already parallel to long axis of the bone. In this type of healing osteoprogenitor cells are from Haversian canals or osteoclastic cutting cone.

In contrast to contact repair, gap repair ensues when there is defects over $0.1 \mathrm{~mm}$, in which the lamellar bones are formed in perpendicular direction to the long axis of the 


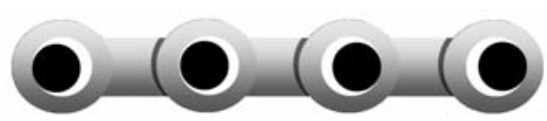

Figure 1. Drill holes in angled eccentric osteosnthesis. Eccentricity of the drill hole away from the fracture gap. The centre of the drill hole is eccentrically positioned away from the fracture gap between both segments.

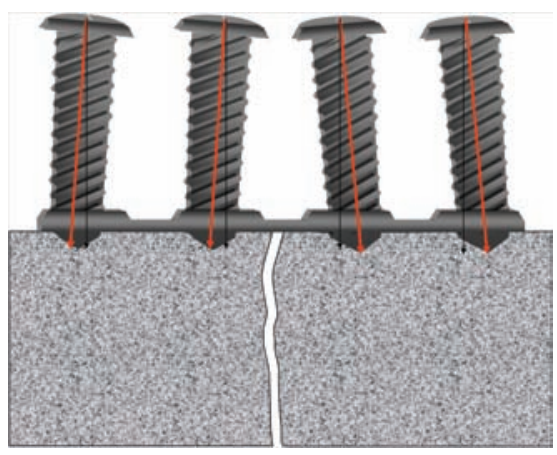

Figure 2. Concept of angled eccentric osteosynthesis. Degree of the drilling instrument to the true perpendicular direction of the bone surface. Because of the gap or break between the outer diameter of a miniscrew and the inner hole of a miniplate, miniscrews can be advanced with some degrees (red arrows) to the true perpendicular direction (black arrows). Advancement of the screws under these conditions (angulation of the drill direction and the eccentric drill hole position away from the centre of the fracture gap) will induce movement of the miniplate to the outer direction, which will produce compressive force of the fragments toward the fracture gap.

bone. Within a period of time they are replaced by those of parallel direction to the long axis as a consequence of remodelling in response to functional loads. If the defect is over $0.2 \mathrm{~mm}$, initial bone is woven bone type. Lamellar bones perpendicular to the long axis of the bone are substituted for the initial woven bone, followed by parallel lamellar bone replacement. Histologically osteoprogenitor cells are from bone marrow in contrast to the

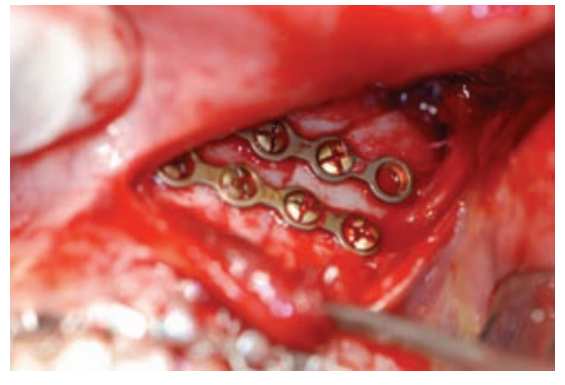

Figure 3. Intraoperative findings showing reduced gap after this technique. Angled eccentric drilling was done followed by an advancement of 2 miniscrews along the drilled hole to reduce the fracture gap originally found on the fracture site.

contact repair in which case the origin of cells is the Haversian canals. ${ }^{8}$

The importance of vascularity on the health of bone during development, growth, and repair was emphasized recently. ${ }^{9}$ Moreover, studies of bone and the immune system is recently uncovering the secrets pertaining to the interplay between the immune system and bone metabolism, converging under the title of osteoimmunology. ${ }^{10}$ The concept of biological internal fixation is advocated today to reduce the sequestration and infection which may be produced by bone necrosis. When we stick to mechanical principles with less emphasis on avoidance of such severe complications, we may lose more important treatment result. In contrast to delayed union or malunion which is more easily managed, the result of bony sequestration is fatal causing the ruin of the patient's quality of life. ${ }^{11}$

Principle of miniplate osteosynthesis accepts monocortical miniscrews according to tension banding principles under the circumstances of simple fracture. Recent A0 principle of the compression osteosynthesis group emphasizes the blood supply to the bone and not disturbing the periosteal vasculatures. Although inferior to contact repair, gap repair is also valuable from the viewpoint of biological internal fixation. Merging the principles of the two schools (miniplate osteosynthesis group and compression osteosynthesis group), I suggest a new technique of angled eccentric osteosynthesis in case of simple mandibular fracture.

\section{References}

1. Perren SM, Russenberger M, Steinemann $\mathrm{S}$, et al. A dynamic compression plate. Acta Orthop Scand Suppl 1969;125:31-41.

2. Allgöwer M, Ehrsam R, Ganz R, et al. Clinical experience with a new compression plate DCP. Acta Orthop Scand Suppl 1969;125:45-61.

3. Niederdellmann H, Akuamoa-Boateng E. Internal fixation of fractures. Int $\mathrm{J}$ Oral Surg 1978;7:252-5.

4. Scolozzi P, Richter M. Treatment of severe mandibular fractures using AO reconstruction plates. Journal of Oral and Maxillofacial Surgery 2003;61:458-61.

5. Hylander WL. Mandibular function in Galago crassicaudatus and Macaca fascicularis: an in vivo approach to stress analysis of the mandible. J Morphol 1979;159: 253-96.

6. Worthington P, Champy M. Monocortical miniplate osteosynthesis. Otolaryngol Clin North Am 1987;20:607-20.

7. Spiessl B. Funktionsstabile Osteosynthese bei Unterkieferfrakturen-Problematik und Technik.[Functionally stable osteosynthesis in mandibular fractures--problems and technic]. Fortschr Kiefer Gesichtschir 1975;19:68-72.

8. Shapiro F. Bone development and its relation to fracture repair. The role of mesenchymal osteoblasts and surface osteoblasts. Eur Cell Mater 2008;15:53-76.

9. Brandi ML, Collin-Osdoby P. Vascular Biology and the Skeleton. Journal of Bone and Mineral Research 2006;21:183-92.

10. Walsh MC, Kim N, Kadono Y, et al. Osteoimmunology: interplay between the immune system and bone metabolism. Annual Review of Immunology 2006;24:33-63.

11. Perren SM. Evolution of the internal fixation of long bone fractures. The scientific basis of biological internal fixation: choosing a new balance between stability and biology. J Bone Joint Surg Br 2002;84: 1093-110. 